\title{
Therapeutic Approaches to Radiation-Induced Fibrosis
}

\author{
Edward J. Macarak, Joel Rosenbloom*
}

The Joan and Joel Rosenbloom Research Center for Fibrotic Diseases, Department of Dermatology and Cutaneous Biology, Sidney Kimmel Medical College, Thomas Jefferson University, Philadelphia, Pennsylvania, USA

\section{Article Info}

\section{Article Notes}

Received: July 10, 2018

Accepted: August 16, 2018

\section{${ }^{*}$ Correspondence:}

Dr. Joel Rosenbloom, MD, PhD, The Joan and Joel Rosenbloom Research Center for Fibrotic Diseases, Department of Dermatology and Cutaneous Biology, Sidney Kimmel Medical College, Thomas Jefferson University, Philadelphia, Pennsylvania, USA; Email: joel.rosenbloom@jefferson.edu.

C 2018 Rosenbloom J. This article is distributed under the terms of the Creative Commons Attribution 4.0 International

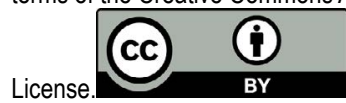

\section{Keywords:}

Fibrosis

Radiation-Induced Fibrosis

Trametinib

Myofibroblast

Therapy

Extracellular Matrix

Fibronectin

\section{ABSTRACT}

Radiation induced fibrosis (RIF) is a common morbidity in patients being treated for cancer with radiation. Off-target effects result in intense inflammatory responses which ultimately results in the generation of extracellular matrix (ECM) producing myofibroblasts which mediate a progressive fibrosis resulting in scarring and organ and tissue dysfunction. Unfortunately, currently, there are no effective therapies to block the excess accumulation of ECM. We have previously reported on the use of trametinib, a MEK inhibitor, to essentially block the formation of abdominal adhesions in a mouse model of cecal abrasion. Using this drug in the mouse model, the complete trans-differentiation of precursor cells into ECM-producing myofibroblasts was blocked. Trametinib is a potentially powerful drug to thwart organ and tissue fibrosis in RIF because it has a potential dual function in that it may block RIF as well as prevent radiation-resistance. Given the intractability of RIF, trametinib should be considered for more extensive testing.

\section{Text}

Radiation induced fibrosis (RIF) is a common morbidity in patients undergoing therapy for tumors. Unfortunately, ionizing radiation not only damages tumor cells but also all other types of cells found within the tumor space as well as in surrounding tissues. Such off-target effects result in an intense inflammatory reaction which initiates cellular responses leading to connective tissue matrix production and intense scar formation. With the arrival of inflammatory cells, a variety of growth factors, the most important and prevalent of which is TGF $\beta$, are activated and/or released from their storage sites within the extracellular matrix (ECM) and from the inflammatory cells themselves. These cytokines mediate an intense fibrotic response largely orchestrated by the myofibroblast cell which dominates the wound healing response in the body. Although its origins are controversial and may vary with the location of the fibrosis, it, nonetheless, is the principle culprit in the uncontrolled generation of scar tissue which alters the structural architecture of the treated area. Unfortunately, the fibrotic response associated with radiation induced fibrosis and other types of fibrosis do not cease with the conclusion of the radiation therapy. The myofibroblasts that are activated remain and continue to produce abnormal amounts of ECM proteins such as collagen, fibronectin and proteoglycans. Under normal circumstances, these excess amounts of ECM would be remodeled by degradative enzymes and return the healing tissue(s) to their normal architectures. In addition, the myofibroblasts which have been responsible for the excess accumulation of ECM would be eliminated either by 
premature senescence, apoptosis or a combination of both. With the disappearance of the myofibroblasts, fibroblast replacements would predominate, returning the tissue to a homeostatic equilibrium of synthesis and degradation with little or no scar formation and additional ECM accumulation. However, in RIF, as in all other fibrotic diseases, this either does not occur or does so at such a slow rate that normal tissue and organ structure is never achieved ${ }^{1}$. Thus, RIF is not an immediate response but rather one which occurs months after the cessation of radiation and may continue for years with the RIF-associated morbidities varying according to the tissues exposed to the radiation. For example, muscle tissue can shorten and atrophy, while skin can harden and joints can become immobile, and frequently all these changes are accompanied by pain and compromised function.

Unfortunately, there are no therapies to block the excess accumulation of ECM and associated scarring and wound contracture. Despite increased understanding of the origin and role of the myofibroblast in RIF and other fibrotic diseases, there are neither drugs which are able to target this cell type specifically nor block its synthetic pathways which become activated and which are responsible for excess ECM production. Likewise, while there are currently successful therapeutic agents which can block the actions of TGF $\beta$, using them might do more harm than good given the wide range of beneficial actions which are mediated by TGF $\beta$.

Despite these rather depressing circumstances, there are promising drugs which may be able to block some, but not all, of the activation pathways associated with profibrotic genes. These drugs, none of which is a "silver bullet" by itself, can be used with one or several other drugs to thwart the pro-fibrotic gene response associated with the initial wound healing response. We have recently reported on the use of a single FDA-approved drug, trametinib, a MEK inhibitor, in a mouse model of cecal abrasion ${ }^{2}$ Using this model, we generated abdominal adhesions in the mouse which initially are comprised of granulation tissue and which subsequently become vascularized and highly cellular resulting in a substantial adhesion between bowel loops. In these experiments, we used three different concentrations of the MEK inhibitor trametinib and found that adhesions were essentially eliminated when the highest dose of trametinib was used.

Our interest in MEK inhibitors for use in blocking abdominal adhesion came as a result of a previous study using rat peritoneal mesothelial cells in which we showed that the mesothelial-mesenchymal transition (MMT) could be blocked by another MEK inhibitor, U0126, a drug not currently in clinical use ${ }^{3}$. When similar studies were carried out using trametinib at 2 and $5 \mathrm{nM}$, the MEK inhibitor blocked the MMT as seen morphologically while
Western analysis showed a dramatic reduction in p-Erk1/2 after stimulation with TGF $\beta 1$ in the presence of either 2 or $5 \mathrm{nM}$ trametinib as did cellular fibronectin containing the EDA isoform ( $\mathrm{FN}^{\mathrm{EDA}}$ ), $\alpha \mathrm{SMA}$, and the $\mathrm{p}$-Smad2 (linker region). These reductions in expression of pro-fibrotic biomarker proteins produced by myofibroblasts strongly suggest that trametinib is interfering with the transition of mesothelial cells into matrix-producing myofibroblasts. Since $\mathrm{Fn}^{\mathrm{EDA}}$ is one of the early biomarkers for the transition of cells into myofibroblasts, we suggest that it may be useful diagnostically to evaluate both the onset of fibrosis and its potential reversal by drug therapy.

The trans-differentiation of precursor cells into myofibroblasts is preceded by the appearance of $\mathrm{Fn}^{\mathrm{EDA}}$. This fibronectin isoform is normally not found in adult tissues but is present during normal wound healing, fibrous tissue formation, and in tumor stroma. While its presence in abdominal fibrosis is novel, its association with myofibroblasts is not. Unfortunately, its molecular role in myofibroblast formation has not been well-characterized. The complete trans-differentiation of a precursor cell into a myofibroblast can be blocked by the IST- 9 anti-Fn ${ }^{\text {EDA }}$ antibody ${ }^{4}$. This antibody is directed against a 10 amino acid epitope encoded within the $\mathrm{Fn}^{\mathrm{EDA}}$ exon which gets spliced into the messenger RNA prior to its being translated ${ }^{5}$. While $\mathrm{Fn}^{\mathrm{EDA}}$ null mice are not embryonic-lethal, such mice have altered wound healing of the skin ${ }^{6}$. Interestingly, Fn ${ }^{\text {EDA }}$ null mice do not exhibit lung fibrosis after being exposed to bleomycin ${ }^{7}$. Since the myofibroblast is the responsible cell in fibrosis, the fact that Fn ${ }^{\mathrm{EDA}}$ null mice cannot develop lung fibrosis indicates that expression of $\mathrm{Fn}^{\mathrm{EDA}}$ is absolutely required for bleomycin-induced lung fibrosis. It has been suggested that an increasingly "hardened" or stiff ECM causes the release of TGF $\beta$ which, in turn, continues to accelerate the formation of myofibroblasts thus intensifying pro-fibrotic changes. However, the actual mechanism of these changes is not clear. It is known that $\mathrm{Fn}^{\mathrm{EDA}}$ binds to specific receptors such as integrins $\alpha 4, \alpha 5$, and $\alpha 9$ as well as TLR4. It is likely that these interactions play a role in governing the behavior of the proto-myofibroblast and myofibroblast as they interact with the matrix in a dynamic equilibrium which will be a fertile ground for future research within the field of fibrosis.

Trametinib is an allosteric MEK inhibitor that was originally developed as an anti-tumor drug, particularly as an anti-melanoma therapeutic agent. In this regard, it has proven moderately successful suggesting it may have wider anti-tumor utility. In many tumors, the MEK pathway is constitutively activated by mutations involving signaling through RAS/RAF leading to activation of Erk1/2 resulting in continuously dividing tumor cells ${ }^{8}$. Therapeutic radiation kills cells by inducing double strand breaks (DSBs) in DNA either by direct photon hits or by the generation of reactive oxygen species leading to cell death 
by one or more mechanisms. Unfortunately, DSBs also subsequently provoke the activation of a large number of cellular signaling pathways which initiate repair processes termed DNA damage response (DDR) which permits tumor cells to thwart the radiation damage and survive the radiation therapy. This occurs because radiation also induces the activation of MEK-Erk and Pi3K-Akt pathways which promote their survival and renders them essentially radio-resistant

As discussed above, the effects of trametinib on fibrotic responses suggested that trametinib might be a useful drug in the amelioration of RIF. However, there has been some concern that when used in combination with radiation therapy, it might interfere with the effectiveness of radiation treatment. This has proven not to be the case. When combined with trametinib, radiation therapy was tested against KRAS-driven pancreatic cancer cells. Trametinib blocked ERK1/2 activation and radiosennsitized multiple mutant lines.. Trametinib delayed resolution of DNA damage and caused repression of both homologous, and non-homologous end-joining, thus circumventing the ability of the tumor cell to become radio-resistant. In addition, trametinib suppressed the expression and activation of several double-strand repair pathway intermediates. Similarly enhanced cytotoxicity was observed in clonogenic survival assays of human BRAF, NRAS, and KRAS-mutant melanoma cell lines. Trametinib also augments the effects of radiation therapy by enhancing stress-induced senescence of tumor cells $s^{9,10}$ In addition, athymic mice administered NRAS-mutant flank melanoma tumors were treated with radiation-therapy and trametinib. Mice receiving the combined treatment showed a reduced mean tumor volume compared to mice receiving trametinib or radiation-therapy alone while the combined administration was well-tolerated ${ }^{8}$. Thus, trametinib has a potential dual function in radiation therapy in that it may block radiation-induced fibrosis and prevent radiationresistance. Given the intractability of RIF, this possibility should be tested without delay.

\section{References}

1. Jun JI Lau LF. Taking aim at the extracellular matrix: CCN proteins as emerging therapeutic targets. Nat Rev Drug Discov. 2011; 10(12): 945-63.

2. Macarak EJ, Lotto CE, Koganti D, et al. Trametinib prevents mesothelial-mesenchymal transition and ameliorates abdominal adhesion formation. J Surg Res. 2018; 227: 198-210.

3. Jin X, Ren S, Macarak E, et al. Pathobiological mechanisms of peritoneal adhesions: The mesenchymal transition of rat peritoneal mesothelial cells induced by TGF-beta 1 and IL- 6 requires activation of Erk1/2 and Smad2 linker region phosphorylation. Matrix Biol. 2016.

4. Hinz B, Celetta G, Tomasek JJ, et al, Alpha-smooth muscle actin expression upregulates fibroblast contractile activity. Mol Biol Cell. 2001; 12(9): 2730-41.

5. Carnemolla B, Borsi L, Zardi L, et al. Localization of the cellularfibronectin-specific epitope recognized by the monoclonal antibody IST-9 using fusion proteins expressed in E. coli. FEBS Lett. 1987; 215(2): 269-73.

6. Muro AF, Chauhan AK, Gajovic S, et al. Regulated splicing of the fibronectin EDA exon is essential for proper skin wound healing and normal lifespan. J Cell Biol. 2003; 162(1): 149-60.

7. Muro AF, Moretti FA, Moore BB, et al. An essential role for fibronectin extra type III domain A in pulmonary fibrosis. Am J Respir Crit Care Med. 2008; 177(6): 638-45.

8. Schick U, Kyula J, Barker $\mathrm{H}$, et al. Trametinib radiosensitises RASand BRAF-mutated melanoma by perturbing cell cycle and inducing senescence. Radiother Oncol. 2015; 117(2): 364-75.

9. Bonner JA, Vroman BT, Christianson TJ, et al. Ionizing radiationinduced MEK and Erk activation does not enhance survival of irradiated human squamous carcinoma cells. Int J Radiat Oncol Biol Phys. 1998; 42(4): 921-5.

10. Estrada-Bernal A, Chatterjee M1, Haque SJ, et al. MEK inhibitor GSK1120212-mediated radiosensitization of pancreatic cancer cells involves inhibition of DNA double-strand break repair pathways. Cell Cycle. 2015; 14(23): 3713-24. 